\title{
Testing the feasibility and safety of the Nintendo Wii gaming console in orthopedic rehabilitation: a pilot randomized controlled study
}

\author{
Andreas Ficklscherer ${ }^{1}$, Jonas Stapf ${ }^{1}$, Kay Michael Meissner ${ }^{1}$, Thomas Niethammer ${ }^{1}$, \\ Matthias Lahner ${ }^{2}$, Markus Wagenhäuser ${ }^{3}$, Peter E. Müller ${ }^{1}$, Matthias F. Pietschmann ${ }^{1}$
}

\author{
${ }^{1}$ Department of Orthopaedic Surgery, University Hospital of Munich (LMU), Munich, \\ Germany \\ ${ }^{2}$ Department of Orthopaedic Surgery, St. Josef-Hospital, Gudrunstrasse, \\ Ruhr-University Bochum, Bochum, Germany \\ ${ }^{3}$ Department of Vascular and Endovascular Surgery, University Hospital Düsseldorf, \\ Düsseldorf, Germany
}

Submitted: 2 August 2014

Accepted: 29 September 2014

Arch Med Sci 2016; 12, 6: 1273-1278

DOI: 10.5114/aoms.2016.59722

Copyright (C 2016 Termedia \& Banach

\section{Abstract}

Introduction: The Nintendo Wii game console is already used as an additional training device for e.g. neurological wards. Still there are limited data available regarding orthopedic rehabilitation. The authors' objective was to examine whether the Nintendo Wii is an appropriate and safe tool in rehabilitation after orthopedic knee surgery.

Material and meethods: A prospective, randomized, controlled study comparing standard physiotherapy vs. standard physiotherapy plus game console training (Wii group) in patients having anterior cruciate ligament $(\mathrm{ACL})$ repair or knee arthroplasty was conducted. The subjects of the Wii group $(n=17$; mean age: $54 \pm 19$ years) performed simple knee exercises daily under the supervision of a physiotherapist in addition to the normal rehabilitation program. The patients of the control group ( $n=13 ; 52 \pm 18$ years) were treated with physiotherapy only. The participants of both groups completed a questionnaire including the International Knee Documentation Committee (IKDC) score, the Modified Cincinnati Rating System and the Tegner Lysholm Knee Score prior to the operation, before discharge from hospital and four weeks after treatment. Results: There was no significant difference in the score results between the Wii and the control group ( $p>0.05)$.

Conclusions: We demonstrated that physiotherapy using the Nintendo Wii gaming console after $\mathrm{ACL}$ reconstruction and knee arthroplasty does not negatively influence outcome. Because training with the Wii device was highly accepted by patients, we see an opportunity whereby additional training with a gaming console for a longer period of time could lead to even better results, regarding the training motivation and the outcome after orthopedic surgery.

Key words: rehabilitation, Nintendo Wii, physiotherapy, orthopedic surgery.

\section{Introduction}

Rehabilitation and recovery of patients after a surgical intervention play a crucial role in orthopedics [1] and take up a lot of time. Nowadays both younger and older patients expect faster rehabilitation and return to familiar mobility.
Corresponding author: Andreas Ficklscherer MD, PhD Department of Orthopaedic Surgery University Hospital of Munich (LMU) Marchioninistr. 15 81377 Munich, Germany Phone: +49 8944000 E-mail: andreas.ficklscherer@ med.uni-muenchen.de 
In order to be able to perform their daily life activities again, many patients are motivated and willing to practice by themselves, even beyond the standard rehabilitation possibilities.

One possibility to enhance the range of standard rehabilitation methods and to enable motivated patients to perform extra practice on their own is virtual reality [2] - a possibility that has usually been associated with high costs and user unfriendly interfaces up until now. Due to wider access to modern, user interactive game consoles, such as the Nintendo Wii, for the first time it is now possible to integrate virtual reality into the patient's rehabilitation process [3].

It allows patients not only to practice on their own, but also gives direct feedback during exercise and motivates the patient to train more in a different way.

There are already numerous rehabilitation institutes and physiotherapy practices which use game consoles as an additional training tool, gaining a lot of patient acceptance [4].

Furthermore, the Nintendo Wii is already used as an evidenced rehabilitation tool in the neurological field for patients who have suffered a stroke. In comparison with conventional post-stroke therapy there is a benefit for arm motor skills of these patients, for example if they played Wii tennis or Wii bowling regularly during inpatient rehabilitation [5]. Another domain within neurological rehabilitation where the Nintendo Wii showed its use as an additional training tool is balance training in patients affected by multiple sclerosis (MS). These patients benefit greatly from home-based balance training by using the additional Nintendo Wii Balance Board, which helps them to perform their daily life activities [6].

Although the Nintendo Wii has shown its potential as a rehabilitation tool within neurology, there is still no academic study which shows a proven benefit of this kind of additional therapy within orthopedics.

There are studies which compared the Wii with conventional rehabilitation methods in functional performance outcomes after hamstring anterior cruciate ligament reconstruction [7] or in the rehabilitation of patients following total knee replacement [8], but all of these studies used original Nintendo gaming software which was not designed for medical use, and none of these studies have shown a significant benefit so far.

In our feasibility/safety study we wanted to pursue a slightly different approach. We used custom made software to examine the suitability of the Nintendo Wii as an additional training tool after orthopedic knee surgery. We wanted to examine whether it is possible to use the Wii for rehabilitation purposes independently of patient age or previous experiences with gaming consoles and without having any negative influences on the healing process. Therefore the study's aim was to examine whether the Nintendo Wii is an appropriate and safe tool in post-surgery rehabilitation. Our hypothesis was that training with the Nintendo Wii does no harm after orthopedic knee surgery in a controlled clinical setting.

This study aims to lay the foundations for further studies which could prove that game consoles are likely to play a prominent role in orthopedic rehabilitation in future and that these devices can complement the present in- and outpatient physiotherapy.

\section{Material and methods}

\section{Participants}

The study received full approval from the Research Ethics Committee (\#280-11). All subjects who participated in the study provided informed consent. Thirty volunteer individuals were divided into two randomized groups and were matched by their age. The inclusion criteria specified patients who had undergone anterior cruciate ligament $(\mathrm{ACL})$ plastic surgery or endoprosthetic surgery of the knee (total knee arthroplasty - TKA).

\section{Nintendo Wii group}

The Wii group consisted of 17 patients, 11 women and 6 men with a mean age of 54 years, who fulfilled the inclusion criteria and who were allowed full weight bearing. This group was treated with standard physiotherapy plus training with the Nintendo Wii. This group included 2 patients undergoing $\mathrm{ACL}$ and 15 patients undergoing TKA surgery.

\section{Control group}

The control consisted of 13 patients, 5 women and 8 men with a mean age of 52 years, who fulfilled the inclusion criteria and were also allowed full weight bearing. The control group was treated with standard physiotherapy alone. This group included 2 patients undergoing $A C L$ and 11 patients undergoing TKA surgery.

\section{Equipment}

The game console was a Nintendo Wii of the newest generation (2012). As the controller we used two Nintendo Wii Remote plus controllers, which measured their position in space via a three-axis accelerometer (ADXL330: SMALL, LOW POWER, 3-AXIS \pm 3 GIMEMS ACCELEROMETER) within one degree of accuracy [9]. The controller and the game console were connected via Bluetooth. 
Kaasa Health (Kaasa Health, Düsseldorf, Germany) developed special, custom made software for our study, which is not available for purchase on the free market. The software was developed using the Nintendo Wii SDK and Adobe's Flash Plug-in for Wii.

The values measured by the controllers were transmitted to the console, which represented the data graphically on the connected monitor.

The following data were displayed on the monitor: maximum and minimum angle, average angle, exercise time and number of knee flexions performed.

\section{Procedures}

After booting and calibrating the Wii controllers, the patients were fitted with two Velcro straps around their legs (Figure 1). One Velcro strap was placed $5 \mathrm{~cm}$ proximal to the knee, the other one $5 \mathrm{~cm}$ proximal to the ipsilateral ankle by the treating physiotherapist.

The patient had to perform simple knee flexion/extension exercises under supervision of the treating physiotherapist. During the whole exercise unit, the patient received graphical feedback via a monitor, connected to the console. Thereby information regarding the time of exercise, the flexion angle and the number of knee flexions was provided. The exercise was performed for $10 \mathrm{~min}$ or until fatigue of the test subject. We daily treated the respective patient group with the Wii as an add-on to the standard physiotherapy, from the first postoperative day until discharge. During the hospital stay every patient was treated for a mean of three therapy sessions.

\section{Data analysis}

Our main outcome measure used in this study for comparing the two groups was the IKDC score [10]. Of secondary importance we also used the Modified Cincinnati Rating System and the Tegner Lysholm Knee Score [11]. Preoperatively, before discharge and 4 weeks after the surgery both groups had to answer a questionnaire including these knee function scores. Additionally the study group had to answer a satisfaction questionnaire at the end of the hospital stay, which contained the following questions:

On a scale from 1 (very poor) to 10 (very good), how would you rate your satisfaction with this study?

Can you imagine using the Wii as a rehabilitation device in physiotherapy practice?

Can you imagine using the Wii as a rehabilitation device (after receiving instructions) at home by yourself?

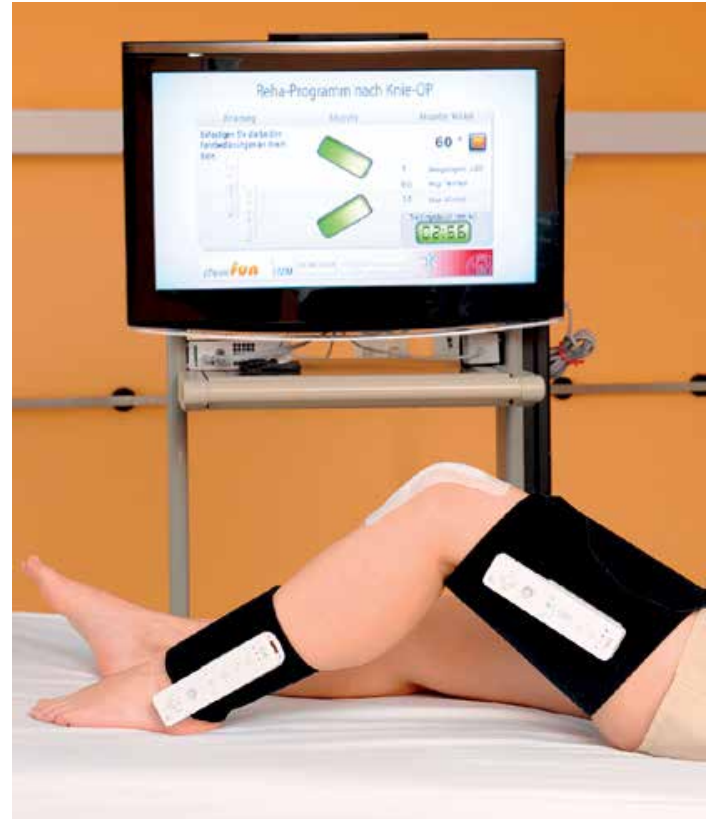

Figure 1. Setup

Does there already exist a Nintendo Wii or a similar game console (Microsoft Xbox, Sony Playstation) at your home?

If not, would you be willing to rent such a rehabilitation device for a small fee for the time of your rehabilitation?

\section{Statistical analysis}

Using Prism 5 (GraphPad Software Inc, Ca, USA) we tested the following null hypothesis $(\mathrm{HO})$ : Median value of the IKDC score of patients treated with standard therapy plus Wii is equal to the median value of the IKDC score of patients treated with standard therapy only.

We compared the postoperative and the 4-week after surgery IKDC results of the Wii group with the same IKDC results of the control group using a two-sided Wilcoxon-Mann-Whitney test for comparing two independent groups. The type I error was set to 0.05 and the power (1-type II error) was set to 0.8. Group size was calculated for 11 patients in each group. We did not perform a power analysis, although this would have been interesting, because our main goal was to prove that the used system/rehabilitation protocol is safe and not harmful.

\section{Results}

After screening for potential candidates for our Wii study, a total of 30 participants were enrolled in our study. Seventeen patients were randomized to the Wii group and 13 patients were randomized to the control group. The average inpatient stay of both groups was 9 days after surgery. Every participant of the Wii group trained $3.2 \pm 1.38$ times 
Table I. Control group knee outcome scores

\begin{tabular}{|lccc|}
\hline Variable & Pre-operative & Post-operative & After 4 weeks \\
\hline IKDC & $28.79 \pm 15.34$ & $28.82 \pm 7.79$ & $39.08 \pm 15.4$ \\
\hline Cincinnati & $33.13 \pm 23.1$ & $35.62 \pm 14.65$ & $43 \pm 19.71$ \\
\hline Tegner Lysholm & $35.41 \pm 25.05$ & $40.62 \pm 18.34$ & $51.25 \pm 24.39$ \\
\hline
\end{tabular}

Table II. Wii group knee outcome scores (means, standard deviation)

\begin{tabular}{|lccc|}
\hline Variable & Pre-operative & Post-operative & After 4 weeks \\
\hline IKDC & $37.48 \pm 16.52$ & $31.16 \pm 13.36$ & $42.66 \pm 19.37$ \\
\hline Cincinnati & $37.42 \pm 16.4$ & $37.18 \pm 18.17$ & $48.56 \pm 22.86$ \\
\hline Tegner Lysholm & $45.58 \pm 20.81$ & $42.53 \pm 22.47$ & $61 \pm 22.72$ \\
\hline
\end{tabular}

with the WII console during their inhospital stay. Patients who underwent ACL surgery trained 2.5 times on average. TKA partients trained 3.1 times during their stay (Tables I and II).

Regarding our main outcome measures, the IKDC score, the Wii group scored $37.48 \pm 16.52$ before and $31.16 \pm 13.36$ points after the Wii therapy. Four weeks after discharge from hospital, the score result improved to $42.66 \pm 19.37$ points when compared to Wii IKDC before discharge. The control group, treated with standard physiotherapy only, scored $28.79 \pm 15.34 ; p=0.076$ before and $28.82 \pm 7.79 ; p=0.45$ points after the inpatient therapy. This result improved 4 weeks after discharge from hospital to $39.08 \pm 15.4$ points; $p=0.67$ (Figure 2).

Regarding our secondary important scores, the Modified Cincinnati Rating System and the Tegner Lysholm Knee Score, the results were similar. Before therapy with the Wii, the Wii group scored $37.42 \pm 16.4$, and after the Wii therapy they scored $37.18 \pm 18.17$ points in the Modified Cincinnati

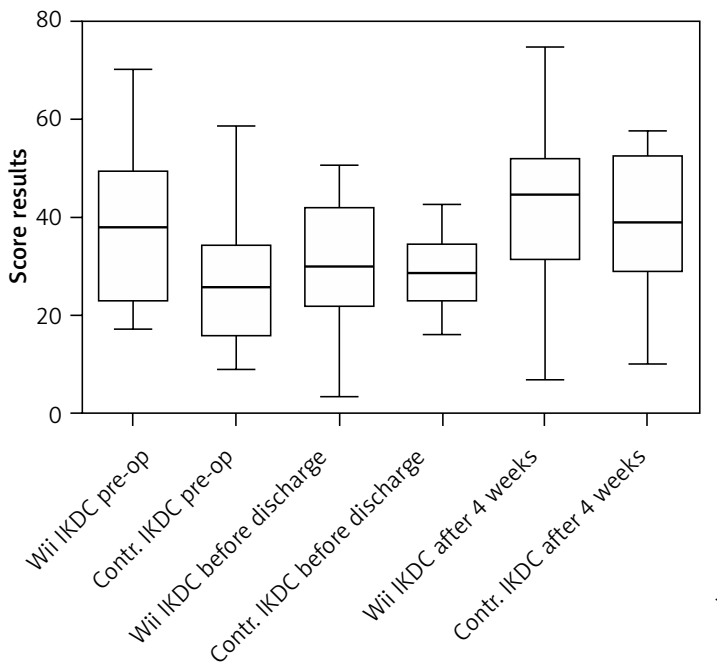

Figure 2. Comparison IKDC score
Rating System. This result improved at 4 weeks after discharge from hospital to $48.56 \pm 22.86$ points. In the same score, the control group scored 33.13 $\pm 23.1 ; p=0.267$ before therapy, $35.62 \pm 14.65$; $p=0.884$ points after therapy and $43 \pm 19.71$; $p=0.596$ points 4 weeks after discharge from hospital (Figure 3).

The Tegner Lysholm Knee Score (Figure 4) also showed a slightly greater improvement of the Wii group. The experimental group scored 45.58 \pm 20.81 before and $42.53 \pm 22.47$ points after therapy sessions with the Wii in the Tegner Lysholm Knee Score. This result improved to $61 \pm 22.72$ points at four weeks after discharge from hospital. The control group only showed an improvement from $35.41 \pm 25.05 ; p=0.12$ to $40.62 \pm 18.34 ; p=$ 0.18 points then to $51.25 \pm 24.39 ; p=0.441$ points in the same period of time.

Even though our results showed a slightly greater improvement of the Wii group we did not find any significant difference between the two groups (Wilcoxon-Mann-Whitney test).

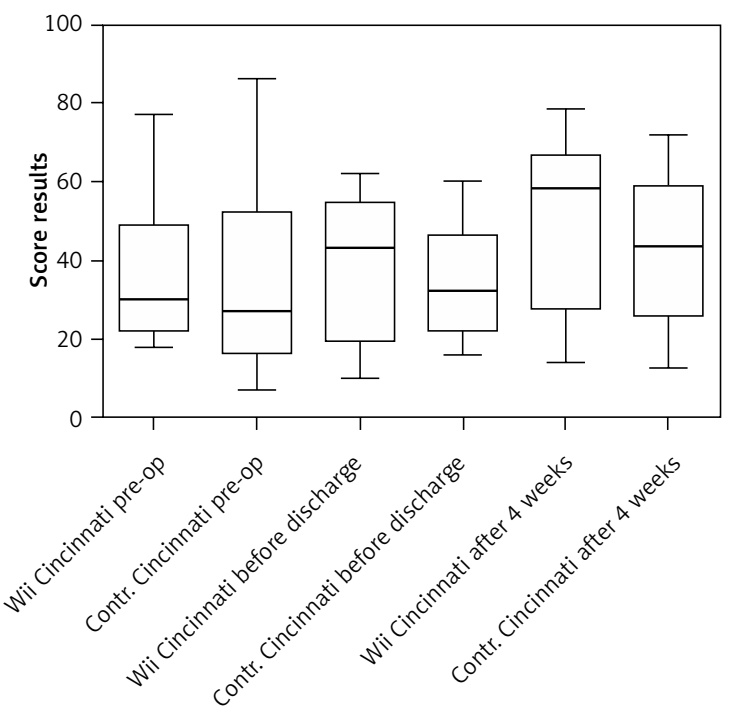

Figure 3. Comparison Modified Cincinnati Rating System 


\section{Discussion}

The study's aim was to examine whether the Nintendo Wii is an appropriate and safe tool in post-surgery rehabilitation. Our hypothesis was that training with the Nintendo Wii does no harm after orthopedic knee surgery in a controlled clinical setting. The main goal of our study was to show that the therapy with the Nintendo Wii using our custom made software is possible and does not affect the patient's healing process in a negative way. Also, we wanted to find out how patients would accept the Wii therapy and how they would get along with the user interface regardless of their age and computer experience. As a consequence of our outcomes this study should lay the foundations for a further home-based therapy with the Wii.

Although we could not prove a significant difference between the Wii group and the control group, we were able to show that a therapy with the Nintendo Wii in a clinical setting, combined with our software, is technically feasible and creates a high amount of motivation, regardless of the different operation methods included in this study or the age of the patient.

As a side effect we could show that the Nintendo Wii is a useful and easy to use device for extending the rehabilitation spectrum and can play an important role in future postoperative knee treatment in hospitals.

This is where we see the main difference to similar studies [7, 8], which also could not demonstrate a significant difference between standard physical therapy and the Wii therapy. All of these studies included only one operation method (total knee replacement or cruciate ligament reconstruction), so that potential positive effects relate exclusively to a relatively small group of patients, who could benefit from this new therapy. In addition, all previous studies used the standard Nintendo Wii Fit software. That means patients had to perform sports such as soccer, bowling, skiing or boxing. In our opinion, the Wii software is at best a fitness software which was not developed for medical purposes and has never been tested. It could bear a risk for potential injuries because of major movements patients had to perform during exercises. That is why we programmed a software which demands only isolated movements of the patients on one movement plane. Due to these differences our above results can be related to a larger group of patients. And with this completely new software the patients are able to perform the exercises in an easier way with reduced risk of injuries.

We see a main reason why we could not show a significant difference between the Wii group and the control group in the short inpatient stay of patients at an average of 9 days after the surgery. Inpatient stay was calculated from the day of

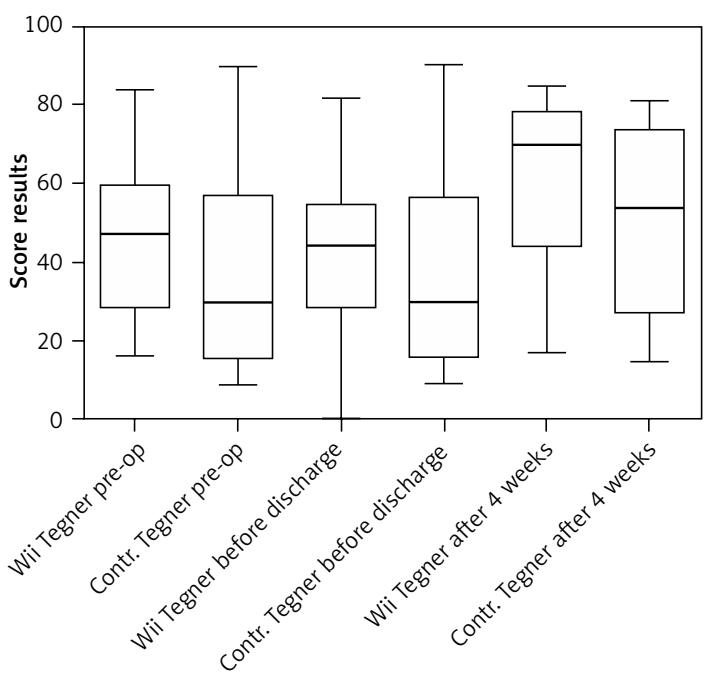

Figure 4. Comparison Tegner Lysholm Knee Score

surgery until their discharge. Most of the patients left in the morning of their discharge without participating in physiotherapy that day. Furthermore, all patients had a wound drainage, the TKA patients usually for 2 days, the ACL patients usually for one day. During that time no physiotherapy was performed. Also there was no physiotherapy at weekends. This all adds up so that in total an average patient had 3 Wii sessions. Probably it takes more practice units to see positive effects of the Wii therapy. Because the above reasons and the study design limited the practice sections, further treatment with the Wii therapy in outpatient rehabilitation facilities or physiotherapy practice after the inpatient stay would be desirable. Furthermore, the used software has to be implemented into a gaming experience to further improve patient motivation (e.g. achieving certain gaming levels, medals). A continuation of the study, to increase the patient cohort, would certainly help to show the benefit of the Wii therapy as well.

We were able to prove that the training with the Wii, after a surgical intervention of the knee, does not affect the healing process in a negative way at all.

In addition, the review of the satisfaction questionnaire at the end of each therapy showed that patients are highly motivated and could imagine continuing the Wii therapy, in addition to the standard therapy, in a physiotherapy practice or even at home.

Although many patients were older and had no prior contact with game consoles or virtual reality, $86.6 \%$ could imagine continuing the therapy with the Wii in a physiotherapy practice or even alone at home $(73.7 \%)$, and $60 \%$ of them would borrow a gaming console for the time of the therapy.

Therefore we are convinced that the acquired motivation and the related increased training of 
patients will have a sustainable positive effect on the ongoing healing process $[12,13]$.

In conclusion, a number of important limitations need to be considered which we were aware of. These known limitations could not be eliminated because of the study design and the fact that our study was planned as a feasibility/safety study. Those limitations will be considered in further studies.

Probably the most important limitation is that the short inpatient stays of patients at an average of 9 days is too short to show the effectiveness of the additional Wii therapy. In further studies, patients will be able to use the Wii gaming console as an outpatient training device in a physical therapy practice or at home, which will give them the possibility to enhance the training sessions.

Second, because of the study design, we were only able to consider for the study patients who had ACL repair or knee arthroplasty. This simultaneously leads to the third limitation, the small patient cohort, which could be easily increased by including other operation methods and longer, possibly outpatient, maintenance.

An advanced study, which eliminates the above limitations, is required to clarify whether our assumptions can be confirmed.

\section{Conflict of interest}

The authors declare no conflict of interest.

\section{References}

1. Heisel J. Rehabilitation following total hip and knee replacement. Der Orthopade 2008; 37: 1217-32.

2. Rizzo A, Kim GJ. A SWOT analysis of the field of virtual reality rehabilitation and therapy. Presence Teleoperators Virtual Environ 2005; 14: 119-46.

3. Deutsch JE, Borbely M, Filler J, Huhn K, Guarrera-Bowlby $P$. Use of a low-cost, commercially available gaming console (Wii) for rehabilitation of an adolescent with cerebral palsy. Phys Ther 2008; 88: 1196-207.

4. Cameirão MS, Badia SB, Oller ED, Verschure P. Neurorehabilitation using the virtual reality based Rehabilitation Gaming System: methodology, design, psychometrics, usability and validation. J Neuroeng Rehabil 2010; 7: 48.

5. Saposnik G, Teasell R, Mamdani M, et al.; Stroke Outcome Research Canada Working G. Effectiveness of virtual reality using Wii gaming technology in stroke rehabilitation: a pilot randomized clinical trial and proof of principle. Stroke 2010; 41: 1477-84.

6. Prosperini L, Fortuna D, Gianni C, Leonardi L, Marchetti MR, Pozzilli C. Home-based balance training using the Wii balance board: a randomized, crossover pilot study in multiple sclerosis. Neurorehabil Neural Repair 2013; 27: 516-25.

7. Baltaci G, Harput G, Haksever B, Ulusoy B, Ozer H. Comparison between Nintendo Wii Fit and conventional rehabilitation on functional performance outcomes after hamstring anterior cruciate ligament reconstruction: prospective, randomized, controlled, double-blind clinical trial. Knee Surg Sports Traumatol Arthrosc 2013; 21: 880-7.
8. Fung V, Ho A, Shaffer J, Chung E, Gomez M. Use of Nintendo Wii Fit in the rehabilitation of outpatients following total knee replacement: a preliminary randomised controlled trial. Physiotherapy 2012; 98: 183-8.

9. Analog Devices I. 2007

10. Higgins LD, Taylor MK, Park D, et al.; International Knee Documentation C. Reliability and validity of the International Knee Documentation Committee (IKDC) Subjective Knee Form. Joint Bone Spine 2007; 74: 594-9.

11. Tegner $Y$, Lysholm J. Rating systems in the evaluation of knee ligament injuries. Clin Orthop Relat Res 1985 198: 43-9.

12. Zimmerli L, Jacky M, Lunenburger L, Riener R, Bolliger $M$. Increasing patient engagement during virtual reality-based motor rehabilitation. Arch Phys Med Rehabil 2013; 94: 1737-46.

13. Moffet H, Collet JP, Shapiro SH, Paradis G, Marquis F, Roy L. Effectiveness of intensive rehabilitation on functional ability and quality of life after first total knee arthroplasty: a single-blind randomized controlled trial. Arch Phys Med Rehabil 2004; 85: 546-56. 